\title{
Convective Transport of Fluid-Solid Interaction: A Study between Non-Newtonian Casson Model with Dust Particles
}

\author{
Abdul Rahman Mohd Kasim 1,*, Nur Syamilah Arifin ${ }^{2}$, Syazwani Mohd Zokri ${ }^{3}$, \\ Mohd Zuki Salleh ${ }^{1}$, Nurul Farahain Mohammad ${ }^{4}$, Dennis Ling Chuan Ching ${ }^{5}$, \\ Sharidan Shafie ${ }^{6}$ (D) and Noor Amalina Nisa Ariffin ${ }^{1}$ \\ 1 Centre for Math. Sci. College of Computing and Applied Sciences, Universiti Malaysia Pahang, Kuantan, \\ Pahang 26300, Malaysia; zuki@ump.edu.my (M.Z.S.); amalinanisa1188@gmail.com (N.A.N.A.) \\ 2 Faculty of Computer and Mathematics Sciences, Universiti Teknologi MARA (UiTM), Cawangan Johor, \\ Kampus Pasir Gudang, Johor 79100, Malaysia; nursyamilaharifin@gmail.com \\ 3 Faculty of Computer and Mathematics Sciences, Universiti Teknologi MARA (UiTM), Cawangan \\ Terengganu, Kampus Kuala Terengganu, Terengganu 21080, Malaysia; syazwanizokri@gmail.com \\ 4 Department of Computational and Theoretical Sciences, IIUM Kuantan, Pahang 25200, Malaysia; \\ farahain@iium.edu.my \\ 5 Fundamental and Applied Science Department, Universiti Teknologi PETRONAS, Perak 32610, Malaysia; \\ dennis.ling@utp.edu.my \\ 6 Department of Mathematical Sciences, Faculty of Science, UTM, Skudai, Johor 79100, Malaysia; \\ sharidan@utm.my \\ * Correspondence: rahmanmohd@ump.edu.my
}

Received: 11 August 2020; Accepted: 10 September 2020; Published: 15 September 2020

\begin{abstract}
The Casson model is a fascinating model, which is genuinely recommended for use with fluids of a non-Newtonian type. The conventional model is not capable to represent the Casson model with the suspension of foreign bodies (dust particles). Due to this, the two-phase model for the mixture of Casson model fluid and dust particles is formulated. This study examines the emerging role of dust particles in changing the behavior of Casson model. In particular, two-phase flow of dusty Casson model with modified magnetic field and buoyancy effect under Newtonian heating boundary condition along a vertically stretching sheet is considered. The equations that govern under Casson model, together with dust particles, are reduced to a system of nonlinear ordinary differential equations by employing the suitable similarity variables. These transformed equations are then solved numerically by implementing the Runge-Kutta-Fehlberg (RKF45) method. The numerical results of skin friction coefficient plus Nusselt number are displayed graphically. The results revealed the fluid's velocity tends to deteriorate due to the existence of dust particles, whilst its temperature is increased. The two-phase flow is one of the mathematical modeling techniques for multiphase flow, where the relationship between the fluid and solid is examined more closely. It is expected that the present findings can contribute to the understanding of the theory of two-phase flow mathematically, which will continue to produce significant research in this field.
\end{abstract}

Keywords: two phase model; Newtonian heating; dusty non-Newtonian fluid; convection transport; modified magnetic field

\section{Introduction}

The convection flow of fluid occurs due to the temperature difference and heat transference rate. In particular, the mechanism of convection can be classified into three types, which are free, forced and 
mixed. Free or natural convection is solely caused by the buoyancy force, while forced convection happens because of the external sources such as pump and fan. Mixed convection occurs when those two convections occur simultaneously. Engineering fields contain many applications of free convection flow, for instance, automatic control system of electrical and electronic components [1]. It is found that the obtained findings from the stretching sheet flow of viscous fluid with free convection are significant for fabric, plastic and polymer industries [2,3]. Therefore, most of the available literature on the flow problem deals with free convection under certain circumstances, such as various types of surfaces, physical parameters, as well as types of fluid and boundary conditions. Together with free convection, the boundary condition of Newtonian heating $(\mathrm{NH})$ has also been conducted by Merkin [4]. As a continuance to this pioneering study, Lesnic et al. [5] and Chaudhary and Jain [6] investigated the viscous fluid flow corresponding to those dimensions. The same problem was also investigated, but with a focus on non-Newtonian fluid flow over a solid sphere, which can be found in [7-9]. In the study reported by Mohamed et al. [10], they showed that the increase in conjugate parameter for $\mathrm{NH}$ has increased the temperature profile of viscous-type fluid. Kasim and Shafie [11] examined the convective flow of viscoelastic-type fluid transient a circular cylinder by taking into account the boundary condition of constant heat flux. The unsteady state of mixed convection flow of micropolar fluid moving along an inclined stretching plate has been scrutinized by Kasim et al. [12]. Furthermore, some interesting studies regarding fluid flow and heat transfer for various aspects have been investigated, as reported in [13-29].

The above literatures have been focused on the investigation of fluid flow only (single phase) and the drawback of this model is that it cannot solve the flow problems of fluids with the suspensions of impurities or dust particles. To overcome this limitation, the two-phase flow model is proposed so that the properties of both fluid and dust particles are taken into account. The packed bed, sedimentation, environmental pollution, centrifugation, purification of crude oil and paint spraying are among the two-phase flow phenomena. The pioneering study reported by Saffman [30] for two-phase flow of gas-carrying solid particles leads to the development of research study in this area. Datta and Mishra [31] investigated the drag and transverse forces on boundary layer flow of dusty viscous fluid. Ramesh et al. [32] investigated the effects of magnetic field and non-uniform source/sink for the stagnation flow of dusty fluid. Manjunatha et al. [33] studied the flow characteristic of dusty fluid over a stretching cylinder in a porosity environment associated with the radiation factor and source of heat. A number of researchers have reported the dusty non-Newtonian fluid flow for various important elements. For instance, Prakash et al. [34] conducted an exact analysis of perturbation technique for dusty viscoelastic fluid in a porous space. Meanwhile, Gireesha et al. [35] focused on the numerical investigation of dusty Maxwell fluid flow with influences of mixed convection, radiation and heating source sources. Recently, Mahanthesh and Gireesha [36] examined the dusty Casson model fluid on the thermal Marangoni convection flow passing a permeable plate. Recently, several works on the dusty non-Newtonian fluid with dissimilar effects have been acknowledged in the literature, as documented in [37-39].

To date, free convection flow with regard to a two-phase flow model under the influence of modified magnetic stimuli has not been discussed in the literature. Therefore, the present problem aims to examine the Casson model with dust particles by introducing the Newtonian heating along the sheet. The model is first transformed into an appropriate form by employing the suitable similarity transformation before applying the Runge-Kutta-Fehlberg (RKF45) for computation. The fluid characteristic correspond to the variations of several physical parameters are illustrated in graphical form.

\section{Problem Formulation}

For this present investigation, the steady, laminar and incompressible two-phase flow of dusty Casson model is considered. The flow of stretching sheet is driven by a uniform stress with the velocity of $u_{w}(x)=a x$. The constant $a$ taking as positive value. Figure 1 illustrates the flow region of a vertical stretching sheet, where an acute angle $\alpha_{1}$ is imposed together with magnetic field. The size as well as 
the densities of the spherical dust are assumed to be the same all over the flow, and it is considered that the particles are non-interacting.

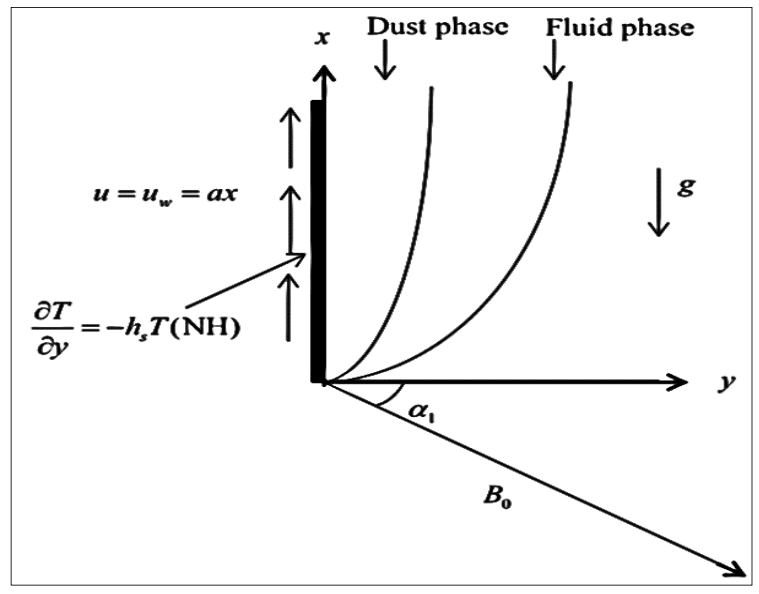

Figure 1. Flow of physical configuration.

The governing equations for steady incompressible dusty Casson model can be expressed by the following equations. Equations (1)-(3) represent the fluid phase, while Equations (4)-(6) regard to dust phase, which can be denoted as $[30,40,41]$

$$
\begin{gathered}
\nabla \cdot \mathbf{V}=0, \\
\rho(\mathbf{V} \cdot \nabla) \mathbf{V}=-\nabla p+\operatorname{div}(\boldsymbol{\tau})+\mathbf{F}_{p}+\mathbf{F}_{b}, \\
\rho c_{p}(\mathbf{V} \cdot \nabla) T=\nabla(k \Delta \cdot \mathbf{T})+Q_{p}, \\
\nabla \cdot \mathbf{V}_{p}=0, \\
\rho_{p}\left(\mathbf{V}_{p} \cdot \nabla\right) \mathbf{V}_{p}=-\nabla p_{p}+\mathbf{F}_{p}, \\
\rho_{p} c_{s}\left(\mathbf{V}_{p} \cdot \nabla\right) T_{p}=-Q_{p},
\end{gathered}
$$

where $\mathbf{V}, T, \rho, \mathbf{F}_{b}, \mathbf{F}_{p}$ and $c_{p}$ are the vector velocity, the fluid temperature, density, pressure, the fluid body force, total fluid-particle interaction force per unit volume and specific heat of fluid at constant pressure, respectively. Meanwhile, $V_{\mathbf{p}}, T_{p}, \rho_{p}$ and $c_{s}$ denote the same properties of dust phase. In Equation (2), div is the divergence operator and $\tau_{i j}$ is the extra stress tensor, in which $\tau_{i j}$ can be expressed as [42]

$$
\tau_{i j}=\left\{\begin{array}{cl}
2\left(\mu_{B}+\frac{\rho_{y}}{\sqrt{2 \pi}}\right) e_{i j}, & \pi>\pi_{c} \\
2\left(\mu_{B}+\frac{\rho_{y}}{\sqrt{2 \pi_{c}}}\right) e_{i j}, & \pi<\pi_{c}
\end{array}\right.
$$

where $\pi=e_{i j} e_{i j}$ is a product deformation component rate for $e_{i j}=1 / 2\left(\partial v_{i} / \partial x_{j}+\partial v_{j} / \partial x_{i}\right)$. The term $\mu_{B}, \pi_{c}$ and $\rho_{y}$ are for plastic dynamic viscosity critical value of product, and yield stress of non-Newtonian fluid.

In order to reduce the complexity of the Casson dusty model, the boundary layer theory and Boussinesq approximation is applied and Equations (1)-(6) can be expressed as follows,

The governing equations for fluid are:

$$
\frac{\partial u}{\partial x}+\frac{\partial v}{\partial y}=0
$$




$$
\begin{aligned}
\rho\left(u \frac{\partial u}{\partial x}+v \frac{\partial u}{\partial y}\right)= & \mu_{B}\left(1+\frac{1}{A}\right)\left(\frac{\partial^{2} u}{\partial y^{2}}\right)+\rho g \beta^{*}\left(T-T_{\infty}\right)+\frac{\rho_{p}}{\tau_{v}}\left(u_{p}-u\right)-\sigma u B_{0}^{2} \sin ^{2} \alpha_{1}, \\
& \rho c_{p}\left(u \frac{\partial T}{\partial x}+v \frac{\partial T}{\partial y}\right)=k\left(\frac{\partial^{2} T}{\partial y^{2}}\right)+\frac{\rho_{p} c_{s}}{\gamma_{T}}\left(T_{p}-T\right),
\end{aligned}
$$

The governing equations for dust are:

$$
\begin{gathered}
\frac{\partial}{\partial x}\left(u_{p}\right)+\frac{\partial}{\partial y}\left(v_{p}\right)=0 \\
\rho_{p}\left(u_{p} \frac{\partial u_{p}}{\partial x}+v_{p} \frac{\partial u_{p}}{\partial y}\right)=\frac{\rho_{p}}{\tau_{v}}\left(u-u_{p}\right), \\
\rho_{p} c_{s}\left(u_{p} \frac{\partial T_{p}}{\partial x}+v_{p} \frac{\partial T_{p}}{\partial y}\right)=-\frac{\rho_{p} c_{s}}{\gamma_{T}}\left(T_{p}-T\right),
\end{gathered}
$$

Note that, the relation between $\gamma_{T}$ and $\tau_{v}$ are generally characterized by $\gamma_{T}=\frac{3}{2} \operatorname{Pr}\left(\frac{c_{s}}{c_{p}}\right) \tau_{v}$.

The governing equations stated above are solved using the following boundary conditions, which are defined as

$$
\begin{gathered}
u=u_{w}(x)=a x, \quad v=0, \quad \frac{\partial T}{\partial y}=-h_{s} T \text { at } y=0 \\
u \rightarrow 0, u_{p} \rightarrow 0, v_{p} \rightarrow v, T \rightarrow T_{\infty}, T_{p} \rightarrow T_{\infty} \text { as } y \rightarrow \infty
\end{gathered}
$$

where $a$ is positive constant. Since the thermal boundary condition Newtonian heating is considered, the term $T$ represents the actual surface temperature and $h_{S}$ is the heat transfer parameter, referring to the situation where the heat transfers initiated from the surface to the surrounding fluid. In addition, at the free stream, the velocity of the dust particles is assumed to approach the fluid velocity while its temperature is conceivably attained at the equilibrium stage.

Equations (8) to (14) are transformed into dimensionless equations using the following similarity transformations, which are given as

$$
\begin{gathered}
u=a x f^{\prime}(\eta), v=-\sqrt{a v} f(\eta), \eta=\sqrt{\frac{a}{v}} y, \theta(\eta)=\frac{T-T_{\infty}}{T_{\infty}} \\
u_{p}=a x F^{\prime}(\eta), v_{p}=-\sqrt{a v} F(\eta), \theta_{p}(\eta)=\frac{T_{p}-T_{\infty}}{T_{\infty}},
\end{gathered}
$$

where $u=\partial \psi / \partial y$ and $v=-\partial \psi / \partial x$, in which $\psi$ represents the stream function. By using Equation (15), the resulting ordinary differential Equations (8)-(13) can be expressed as

$$
\begin{gathered}
\left(1+\frac{1}{A}\right) f^{\prime \prime \prime}(\eta)+f(\eta) f^{\prime \prime}(\eta)-\left(f^{\prime}(\eta)\right)^{2}+\operatorname{Gr} \theta(\eta)+\beta N\left(F^{\prime}(\eta)-f^{\prime}(\eta)\right) \\
-M \sin ^{2} \alpha_{1} f^{\prime}(\eta)=0 \\
\theta^{\prime \prime}(\eta)+\operatorname{Pr} f(\eta) \theta^{\prime}(\eta)+\frac{2}{3} \beta N\left(\theta_{p}(\eta)-\theta(\eta)\right)=0 \\
\left(F^{\prime}(\eta)\right)^{2}-F(\eta) F^{\prime \prime}(\eta)+\beta\left(F^{\prime}(\eta)-f^{\prime}(\eta)\right)=0 \\
\theta_{p}^{\prime}(\eta) F(\eta)+\frac{2}{3} \frac{\beta}{\operatorname{Pr} \lambda}\left(\theta(\eta)-\theta_{p}(\eta)\right)=0
\end{gathered}
$$

The transformed boundary conditions (14) are denoted as

$$
\begin{gathered}
f(0)=0, f^{\prime}(0)=1, \theta^{\prime}(0)=-b(1+\theta(0)) \text { as } \eta=0 \\
f^{\prime}(\eta) \rightarrow 0, F(\eta) \rightarrow 0, F(\eta) \rightarrow f(\eta), \theta(\eta) \rightarrow 0, \theta_{p}(\eta) \rightarrow 0 \text { at } \eta \rightarrow \infty
\end{gathered}
$$

where ()$^{\prime}$ is the first derivatives of any function with respect to $\eta$. The dimensionless parameters contained in Equations (16)-(20) are defined as 
Mass concentration of particle phase:

$$
N=\rho_{p} / \rho
$$

Magnetic field:

$$
M=\sigma B_{0}^{2} / \rho a
$$

Fluid-particle interaction:

$$
\beta=1 / a \tau_{v}
$$

Prandtl number:

$$
\operatorname{Pr}=\mu c_{p} / k
$$

Specific heat ratio of mixture:

$$
\lambda=c_{s} / c_{p}
$$

Grashof number:

$$
\mathrm{Gr}=g \beta^{*} T_{\infty} / b^{2} x
$$

Conjugate parameter for $\mathrm{NH}$ :

$$
b=-h_{s}(v / a)^{1 / 2}
$$

Here the $\mathrm{Gr}=g \beta^{*} T_{\infty} / b^{2} x$ is the local Grashof number and is still in the function of $x$. It is worth mentioning that, Equations (16)-(19) are required for the similarity transformation as represented in Equation (15), whereby the solutions should solely depend on variable $\eta$. Thus, variable $x$ that appears in Gr needs to be eliminated so that it can be considered as a constant. Subsequently, Gr can be redefined by assuming $\beta^{*}=b x$, where $\beta^{*}$ has the dimension of $x$ (length) and $b$ is a constant value. Since the term $x$ only happened to be in the local Grashof number parameter, the particular relations are anticipated to make the obtained equations more realistic. The parameter $\mathrm{Gr}$ can now be written as $\mathrm{Gr}=g T_{\infty} / b$. A comprehensive explanation of this approach was documented by Makinde [43] and Gangadhar et al. [44] and recently applied by Seshadri and Munjam [45] in their problem formulation.

The forced convection flows of non-Newtonian fluid for Casson model can be retrieved from this study, by directly substituting $\beta=N=M=0$ and $\gamma \rightarrow \infty$ in Equation (16). Thus, this flow case can be treated as the limiting case, which arises from this present study. As referred to Mukhopadhyay [46], the exact solution for this limiting case is denoted by

$$
f(\eta)=\sqrt{1+\frac{1}{A}}\left(1-\exp \left(-\frac{\eta}{(1+1 / A)^{1 / 2}}\right)\right)
$$

Subsequently, the analytical solution of $\theta(\eta)$ can be determined by using the following expression

$$
\theta(\eta)=C_{1} \int_{\eta}^{\infty} e^{-\operatorname{Pr} \int_{\eta}^{\infty} f d \eta} d \eta, C_{1}=b(1-\theta(0)) / \exp \left(-\operatorname{Pr} \int_{\eta}^{\infty} f d \eta\right)
$$

The fluid near to the sheet surface is dragged when the fluid starts moving along it, which then actuates the friction force that behaves opposite to the flow direction. Together with that, heat begins to transfer within fluid and stretching sheet. In this model, the skin friction coefficient $C_{f}$ and local Nusselt number $\mathrm{Nu}_{x}$ are measured. These physical quantities are, respectively, evaluated using, $C_{f}=\frac{\tau_{w}}{\rho U_{w}^{2}(x)}$, and $\mathrm{Nu}_{x}=\frac{x q_{w}}{k\left(T_{w}-T_{\infty}\right)}$ where $\tau_{w}=\left(\mu_{B}+\frac{\rho_{y}}{\sqrt{2 \pi_{c}}}\right)\left(\frac{\partial u}{\partial y}\right)_{y=0}$ and $q_{w}=-k\left(\frac{\partial T}{\partial y}\right)_{y=0}$. Utilizing all the previously mentioned terms, the physical quantities of skin friction coefficient $C_{f}$ are estimated as

$$
C_{f} \operatorname{Re}_{x}^{1 / 2}=\left(1+\frac{1}{A}\right) f^{\prime \prime}(0),
$$


and the local Nusselt number $\mathrm{Nu}_{x}$ is predicted as

$$
\mathrm{Nu}_{x} \operatorname{Re}_{x}^{-1 / 2}=b\left(1+\frac{1}{\theta(0)}\right)
$$

where $\operatorname{Re}_{x}=\left(a x^{2} / v\right)$ declared as a Local Reynolds number.

\section{Numerical Procedure}

Among the various numerical methods, the Runge-Kutta-Fehlberg (RKF45) method is employed in this study because of its undeniable success for solving the two-phase flow problems, which can be found in many existing works such as reported by Isa et al. [47] and Ramesh et al. [48]. Thus, it is believed that this method is very stable and relevant for this kind of problem. In order to obtain solutions, Equations (16)-(19) along with the boundary conditions of (20) are solved by using the RKF45 method, and the numerical computation is carried out using Maple software. The numerical solutions obtained from this study are needed to satisfy the boundary conditions (20) to confirm their correctness. Therefore, the suitable finite value of $\eta_{\infty}=8$ is chosen since the velocity and temperature profiles are asymptotically approaching zero at free stream. The whole computation has used the default relative error as $10^{-6}$, while the final grid size and relative error at the end of estimation process were use the value of 0.02 and $10^{-4}$, respectively.

\section{Results and Discussion}

The dusty Casson model on free convection flow, with the effect of an aligned magnetic field subjected to boundary conditions of $\mathrm{NH}$, has been presented. The significant feature of this model is that the governing equations for each phase are formulated separately, and they are coupled via an interaction term. It is also worth noting that if $\beta=N=0$ and $\alpha_{1}=\pi / 2$ is substituted into Equations (16)-(19), this present study replaces the free convection of the Casson model flow problem with a transverse magnetic field. To assure the accuracy, the present numerical results are compared with published work as reported by Vajravelu et al. [49] and exact equation (21) illustrated in Table 1. In their study, an optimal homotopy analysis method (OHAM) has been used to obtain the results of $-f^{\prime \prime}(0)$, specifically for the case of linear stretching sheet of Casson model without mixed convection effects. As shown in the table, those results agreed reasonably well, which validates the obtained present solutions. Note that, the computed results from the current study as presented in Table 1 are acquired by completely ignoring the parameters $\beta, N, \mathrm{Gr}, M$. The numerical analyses of flow behavior and heat transfer process are then performed with regard to the various values of controlling parameters. The fixed values of parameter $\operatorname{Pr}=0.72, \alpha_{1}=\pi / 6, \beta=0.5, N=0.5, M=1, A=1, \mathrm{Gr}=0.1, \lambda=0.1$ and $b=0.2$ are computed throughout the study except from the variation of their values as illustrated in the Figures $2-19$.

Table 1. Numerical values of $-f^{\prime \prime}(0)$ when $\beta=N=\mathrm{Gr}=M=0$.

\begin{tabular}{cccc}
\hline $\boldsymbol{A}$ & Exact Equation (21) & Vajravelu et al. [49] & Present \\
\hline$\infty$ & 1.0000 & 1.0000 & 1.0000 \\
1 & 0.707107 & 0.707107 & 0.707107 \\
2 & 0.816497 & 0.816497 & 0.816497 \\
3 & 0.866025 & 0.866025 & 0.866025 \\
4 & 0.894427 & 0.866025 & 0.894427 \\
\hline
\end{tabular}




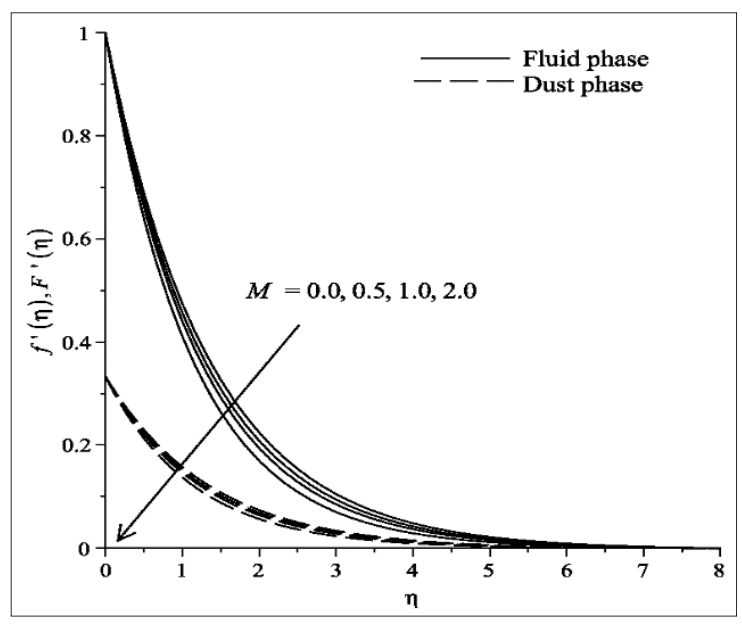

Figure 2. Profile on velocity for various $M$.

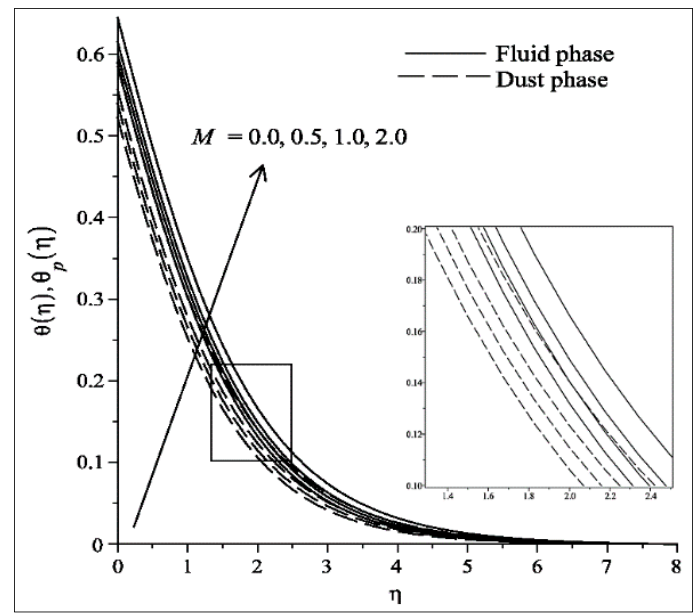

Figure 3. Profile on temperature for various $M$.

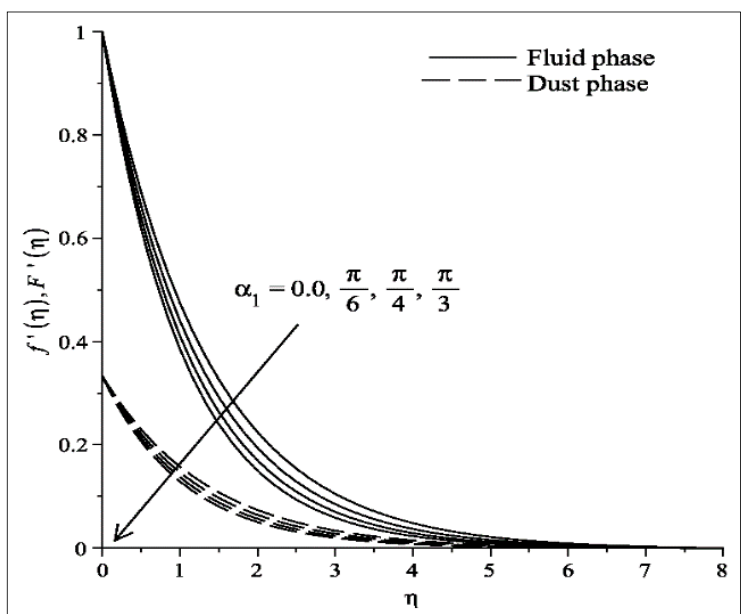

Figure 4. Profile on velocity for various $\alpha_{1}$. 


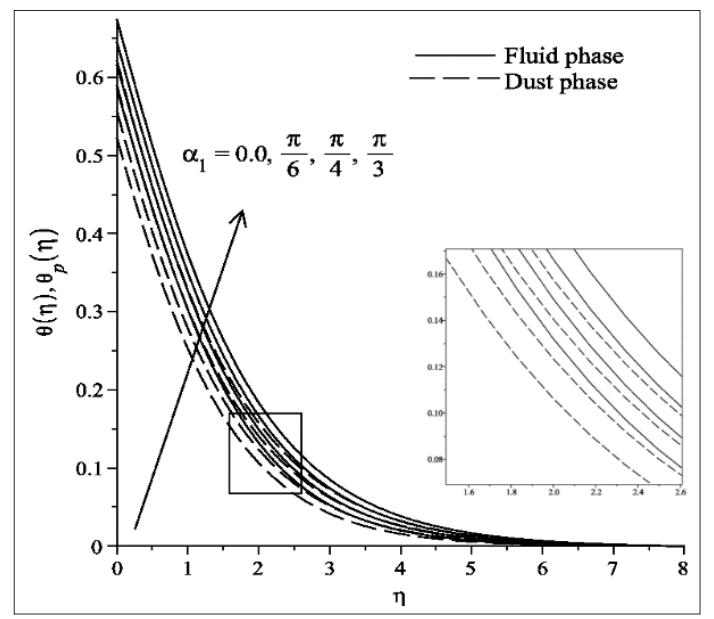

Figure 5. Profile on temperature for various $\alpha_{1}$.

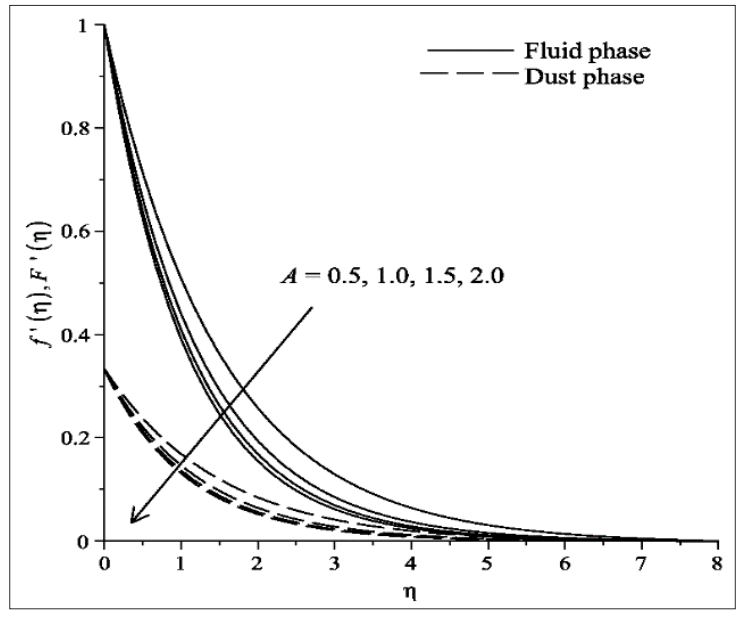

Figure 6. Profile on velocity for various $A$.

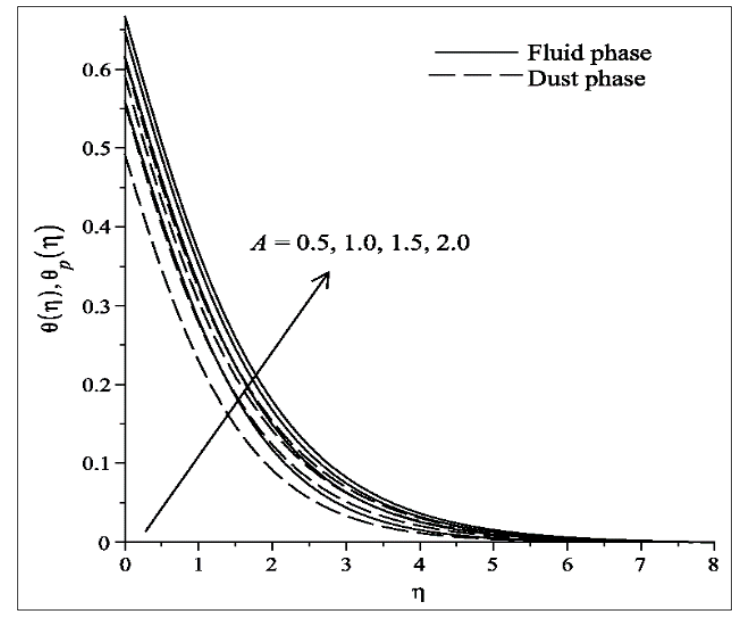

Figure 7. Profile on temperature for various $A$. 


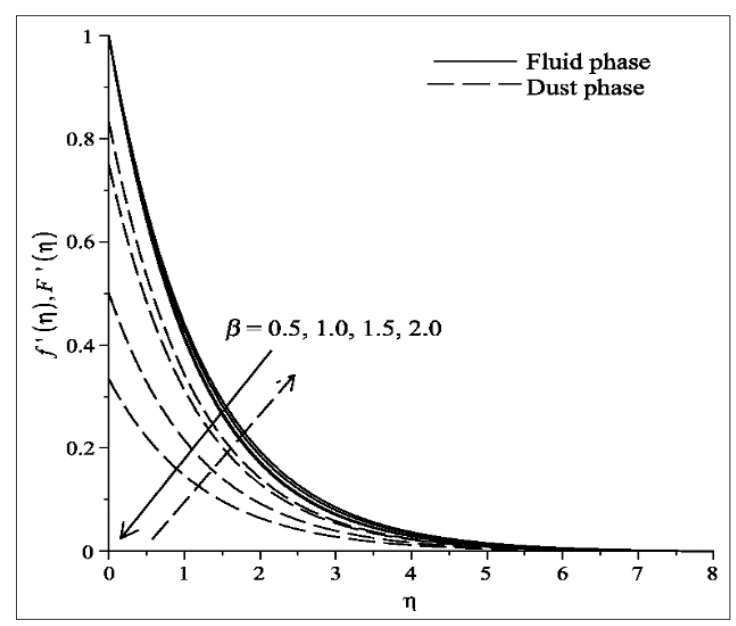

Figure 8. Profile on velocity for various $\beta$.

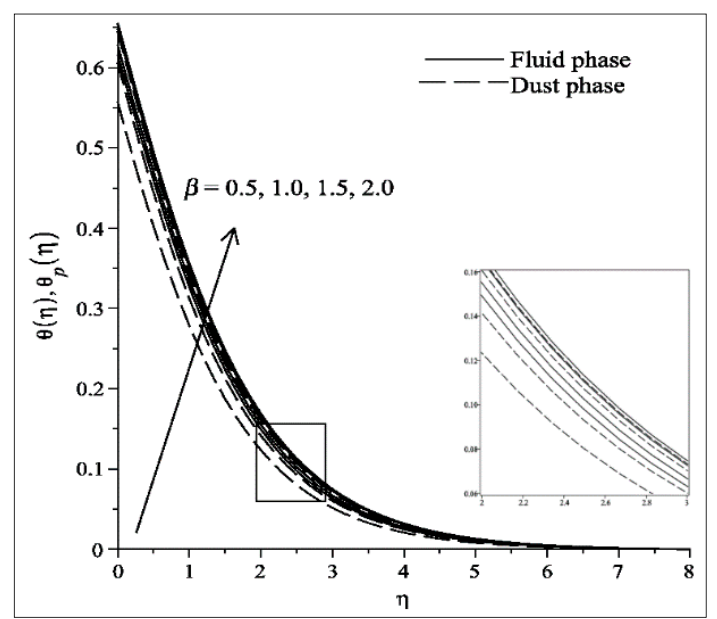

Figure 9. Profile on temperature for various $\beta$.

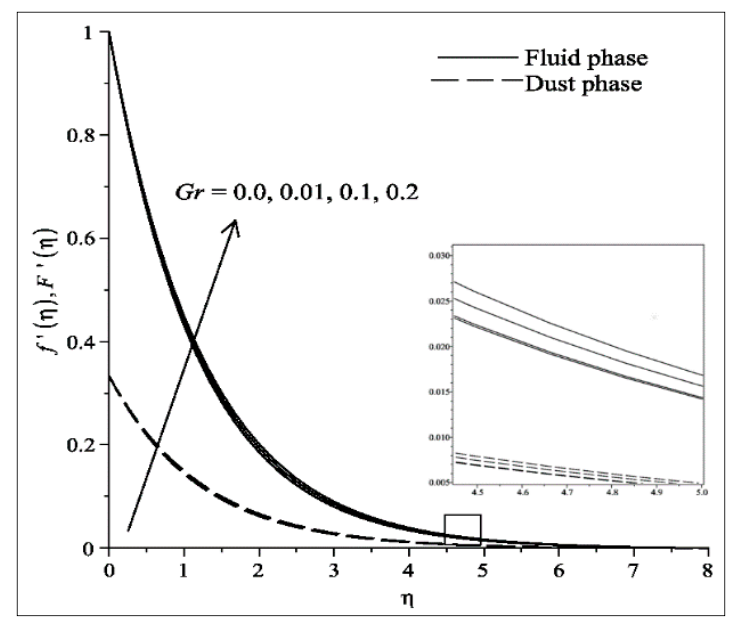

Figure 10. Profile on velocity for various $G r$. 
Crystals 2020, 10, 814

10 of 18

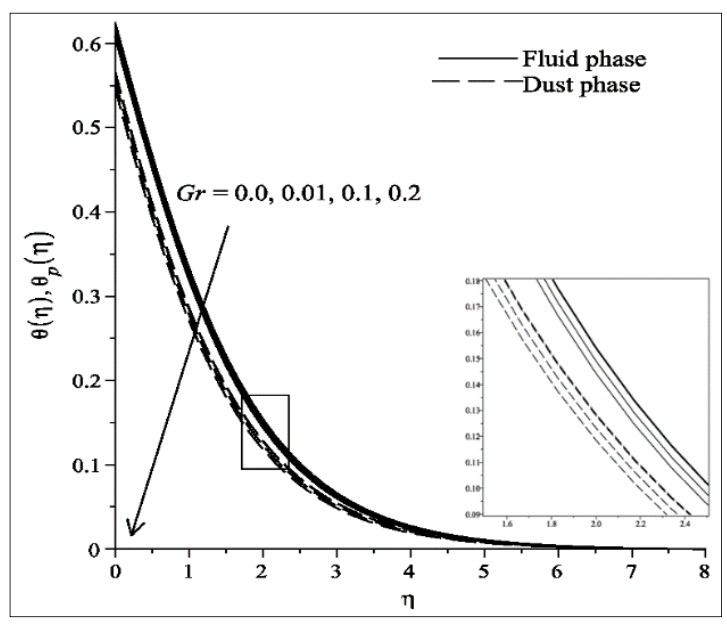

Figure 11. Profile on temperature for various $G r$.

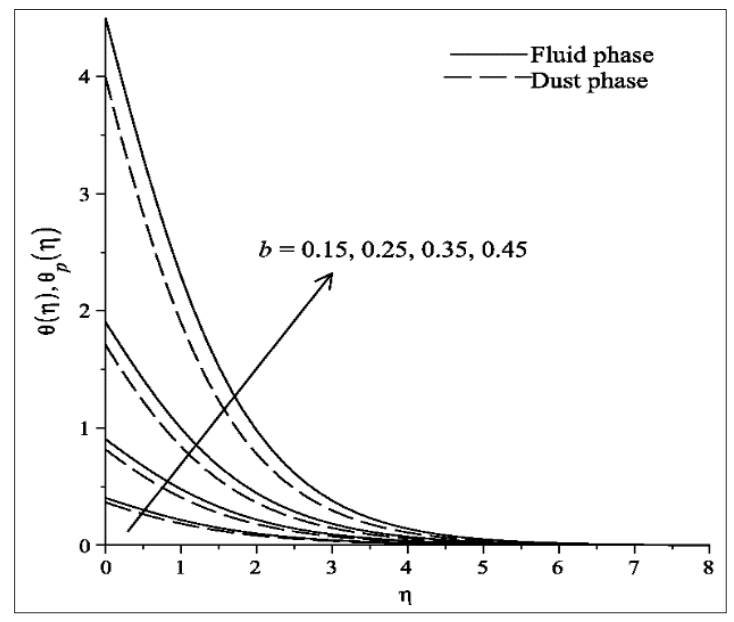

Figure 12. Profile on temperature for various $b$.

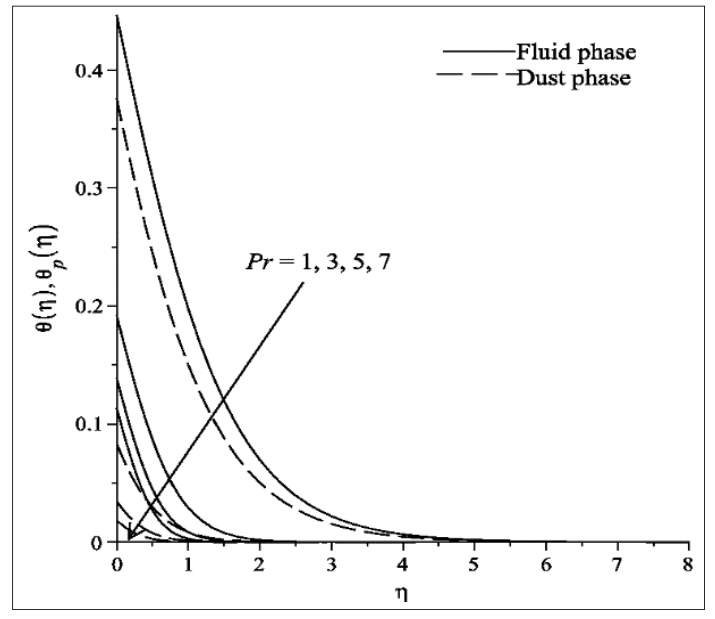

Figure 13. Profile on temperature for various Pr. 


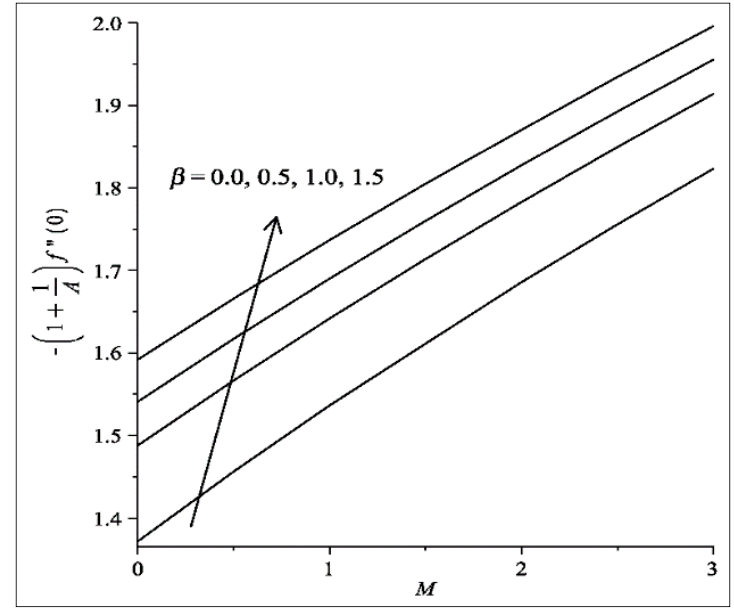

Figure 14. Various values of $\beta$ on the skin friction coefficient and $M$.

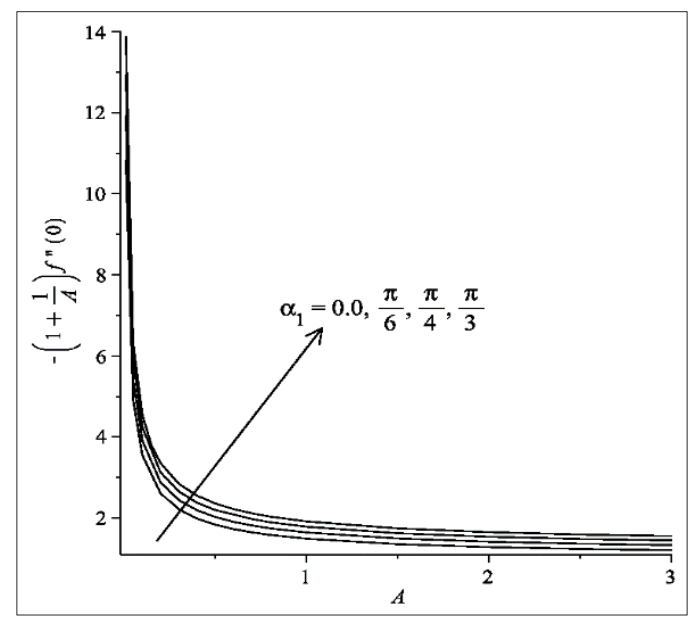

Figure 15. Various values of $\alpha_{1}$ on the skin friction coefficient and $A$.

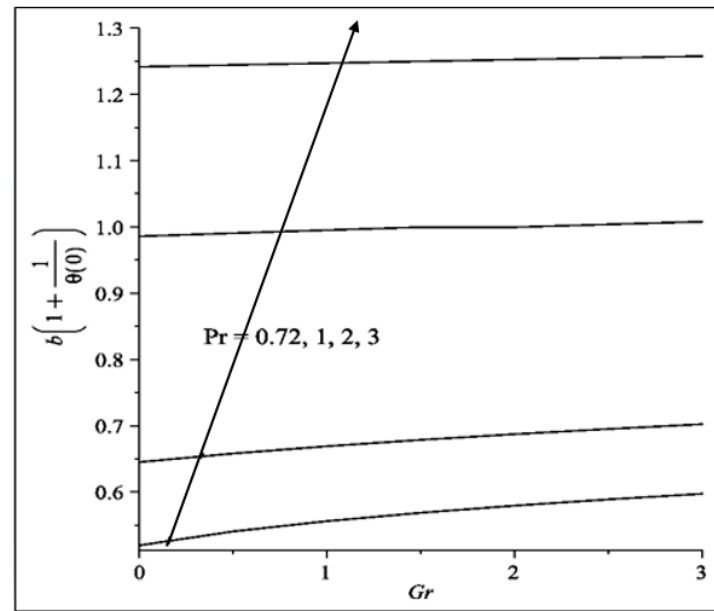

Figure 16. Various values of Pr on Nusselt number and Gr. 


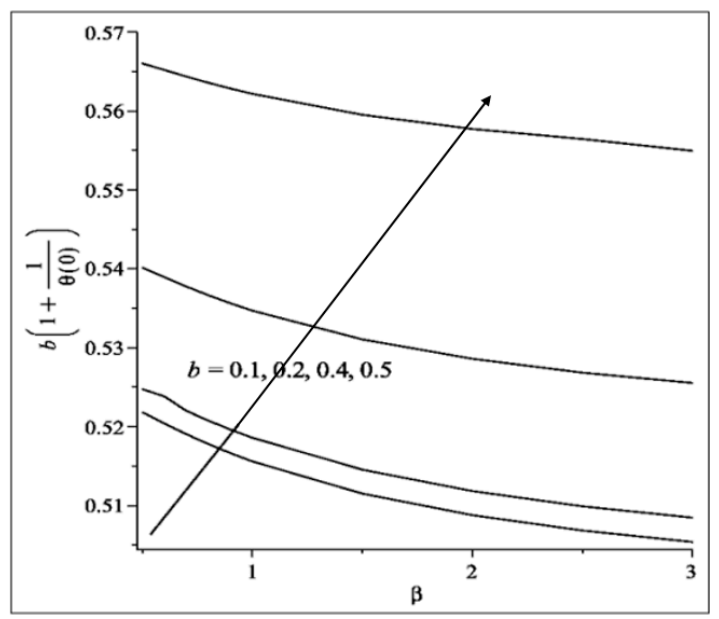

Figure 17. Various values of $b$ on Nusselt number and $\beta$.

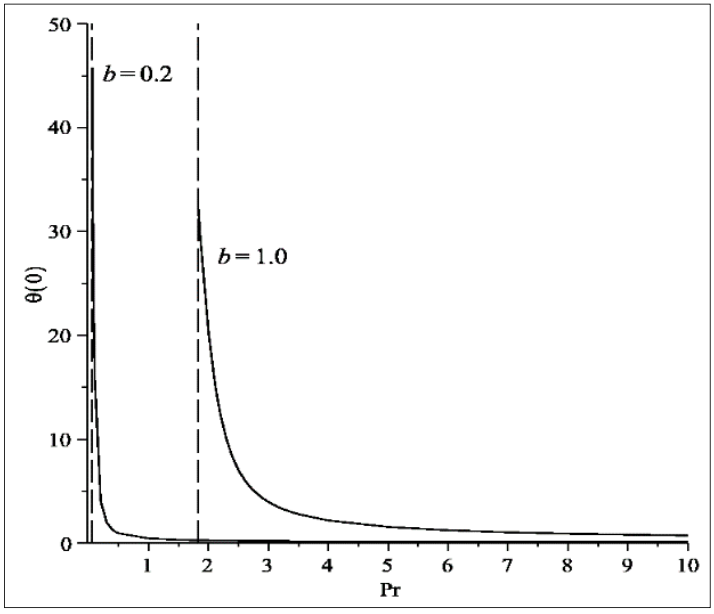

Figure 18. Various values of $b$ on surface temperature and Pr.

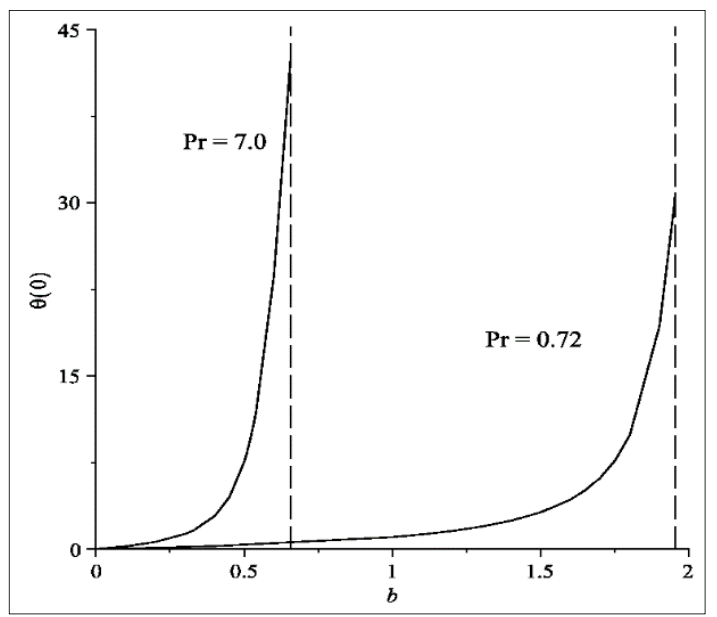

Figure 19. Various values of $\operatorname{Pr}$ on surface temperature and $b$.

Table 2 presents the numerical values of skin friction coefficient, $-f^{\prime \prime}(0)$ and temperature, $\theta(0)$ of the fluid flow at the surface of the sheet by varying the values of controlling parameters. It can be noticed that, the magnitude values of $f^{\prime \prime}(0)$ decreases when $\mathrm{Gr}$ and $b$ are increased, while its magnitude value is found to increase as the remaining parameters escalate. On the other hand, the value of $\theta(0)$ 
reduces with the growing effects in Gr and Pr but increases upon the increase of other parameters. From the results demonstrated in this table, one can expect that the increasing value of $f^{\prime \prime}(0)$ led to the deceleration of fluid motion of the corresponding parameters as a consequence of high friction occurring on the surface. However, a similar trend between $\theta(0)$ and the temperature profile of the fluid phase is observed for all investigated parameters. Therefore, to provide a better picture of those observations, the profiles of velocity and temperature of the fluid phase under the influence of the involved parameters will be depicted in the ensuing figures. Note that, the negative sign of $f^{\prime \prime}(0)$ is associated with the dragging force generated by the surface on the fluid.

Table 2. Numerical values of $-f^{\prime \prime}(0)$ and $\theta(0)$ for various values of parameters.

\begin{tabular}{|c|c|c|c|c|c|c|c|c|}
\hline$A$ & $\alpha_{1}$ & $M$ & $\beta$ & Gr & $b$ & Pr & $-f^{\prime \prime}(0)$ & $\boldsymbol{\theta}(0)$ \\
\hline 0.5 & $\pi / 6$ & 1 & 0.5 & 0.1 & 0.2 & 0.72 & 0.67448 & 0.55931 \\
\hline 1 & & & & & & & 0.82101 & 0.61584 \\
\hline 1.5 & & & & & & & 0.89639 & 0.64724 \\
\hline 2 & & & & & & & 0.94288 & 0.66730 \\
\hline \multirow[t]{4}{*}{1} & 0 & 1 & 0.5 & 0.1 & 0.2 & 0.72 & 0.74410 & 0.58564 \\
\hline & $\pi / 6$ & & & & & & 0.82101 & 0.61584 \\
\hline & $\pi / 4$ & & & & & & 0.89159 & 0.64544 \\
\hline & $\pi / 3$ & & & & & & 0.95714 & 0.67457 \\
\hline \multirow[t]{4}{*}{1} & $\pi / 6$ & 0 & 0.5 & 0.1 & 0.2 & 0.72 & 0.74409 & 0.58564 \\
\hline & & 1 & & & & & 0.78344 & 0.60082 \\
\hline & & 1.5 & & & & & 0.82101 & 0.61584 \\
\hline & & 2 & & & & & 0.85700 & 0.63071 \\
\hline \multirow[t]{4}{*}{1} & $\pi / 6$ & 1 & 0.5 & 0.1 & 0.2 & 0.72 & 0.82101 & 0.61584 \\
\hline & & & 1 & & & & 0.84512 & 0.62775 \\
\hline & & & 1.5 & & & & 0.85922 & 0.63581 \\
\hline & & & 2 & & & & 0.86848 & 0.64135 \\
\hline \multirow[t]{4}{*}{1} & $\pi / 6$ & 1 & 0.5 & 0 & 0.2 & 0.72 & 0.84172 & 0.62508 \\
\hline & & & & 0.01 & & & 0.83961 & 0.62411 \\
\hline & & & & 0.1 & & & 0.82101 & 0.61584 \\
\hline & & & & 0.2 & & & 0.80118 & 0.60758 \\
\hline \multirow[t]{4}{*}{1} & $\pi / 6$ & 1 & 0.5 & 0.1 & 0.15 & 0.72 & 0.82816 & 0.40201 \\
\hline & & & & & 0.25 & & 0.81147 & 0.90284 \\
\hline & & & & & 0.35 & & 0.77880 & 1.90256 \\
\hline & & & & & 0.45 & & 0.69712 & 4.49964 \\
\hline \multirow[t]{4}{*}{1} & $\pi / 6$ & 1 & 0.5 & 0.1 & 0.2 & 1 & 0.82852 & 0.44655 \\
\hline & & & & & & 3 & 0.83811 & 0.19196 \\
\hline & & & & & & 5 & 0.83962 & 0.13876 \\
\hline & & & & & & 7 & 0.84024 & 0.11334 \\
\hline
\end{tabular}

Figures 2-5 display the velocity profile of fluid and dust phases with regard to the parameters of magnetic field, $M$ and aligned angle, $\alpha_{1}$. It can be observed from those figures that, the velocity profiles are reduced when both parameters increased. The growth of effect of $M$ is solely caused by the increment of $\alpha_{1}$ and the correlation between two parameters can be mathematically explained through Equation (16). Therefore, the drag (Lorentz) force has been developed on the flow region and caused the motion of fluid and dust particles to decelerate. In addition, the present problem can be reduced to a free convection flow problem of a dusty Casson model in the absence of a magnetic field if $\alpha_{1}=M=0$, while $\alpha_{1}=\pi / 2$ corresponds to the transverse magnetic field flow.

Figures 6 and 7 portray the variation values of Casson parameter, $A$ on the velocity and temperature profiles. As illustrated in Figure 6, the increase in value of $A$ leads to the decrease in the velocity profile of the fluid phase, since the yield stress became lower to cause a situation in which the fluid behaves like a rigid body. Thus, the transport rate of the fluid is depreciated. It can also be noticed that the 
motion of dust particles is decelerated. Meanwhile, this observation is opposite to the temperature profile, in which the increasing trend is noticed. It is worth mentioning that, as $A \rightarrow \infty$, the Casson model reduces to Newtonian model.

Figures 8 and 9 exhibit the variation values of parameter $\beta$, in which it is evaluated to analyse the influence of dust particles in the Casson model. Note that, when $\beta=0$, the single phase flow of Casson model without the presence of dust particles can be recovered from this present problem. As referred to Siddiqa et al. [41], dust particles begin to move once the fluid flows. It can be seen in Figure 8 that the velocity profile of the fluid (dust) phase decrease (increase) as the value of $\beta$ is increased. This is due to the decrease in relaxation time of particle phase $\tau_{v}$, where the dust particles adjust its velocity and move quickly through the fluid carrier so that the velocities of both fluid and dust particles will be equilibrium. On the other hand, the temperature profile of all phases are observed to increase, and it can be see that the variation of $\beta$ insignificantly affect the temperature profile as compared to the velocity profile.

Figures 10 and 11 demonstrate the influence of buoyancy intensity on the dusty Casson model by evaluating the Grashof number, Gr. A slight increase in velocity profile is noticed as Gr increased. Physically, the influence of increasing buoyancy force results in a rise in temperature differences, causing the fluid motion to accelerate. Conversely, the temperature profile of both phases reduces as $\mathrm{Gr}$ is increased. It can be seen from those figures that, the variation of both profiles show a small difference when the value of $\mathrm{Gr}$ changed.

Figure 12 shows that the increasing impact of conjugate parameter $b$ results in the increase in temperature profile for both phases. The possible explanation stemming from this might be that, for a large value of $b$, the heat transfer parameter $h_{s}$ enhances, and thus encourages the heat transfer from the heated surface to fluid more rapidly. In contrast, the increasing effect of Pr reduces the temperature profile of both phase, as shown in Figure 13. From the theoretical perspective, the higher value of $\mathrm{Pr}$ has a low thermal conductivity, and correspondingly reduced the capability of fluid to absorb heat from the heated sheet.

Figure 14 elucidates the increasing value of magnitude value of skin friction coefficient with $\beta$ as $M$ is increased. However, the decreasing trend of magnitude value of skin friction coefficient with regard to the increase in values of $\alpha_{1}$ and $A$ is observed, as shown in Figure 15. It could be attributed to the augmentation of magnetic field strength, which gives rise to the increase in drag-like force on the flow regime. Because of this, the fluid flow tends to retard and it is expected that, the velocity of fluid decelerates, as shown in Figures 2, 4, 6 and 8. The negative value of skin friction coefficient reflects the dragging force caused by the sheet to the fluid [46].

Figures 16 and 17 provide the correlation of $\mathrm{Gr}$ and $\beta$ with various values of $\operatorname{Pr}$ and $b$, respectively on the Nusselt number. Both parameters of Pr and $b$ are important in the convection process in the heat transfer problem, since they have significantly influenced the temperature profile, which can be seen in Figures 12 and 13. Therefore, in those figures, a noticeable change in the variation of Nusselt number is observed. From Figure 16, the increase in value of Pr as Gr is enhanced can be seen and this tends to increase the Nusselt number. Meanwhile the decreasing trend of Nusselt number corresponds to the increase in values of $b$ and $\beta$ is observed, as shown in Figure 17. These observations are expected, since the fluid temperature at the surface is inversely proportional to Nusselt number, as denoted in Equation (24). As a result, the decrease in value of surface temperature leads to the increase in Nusselt number. Moreover, this implies to the decreasing (increasing) trends of fluid temperature with regard to the enhancement effects of $\operatorname{Pr}$ and $\mathrm{Gr}(b$ and $\beta)$, as shown in Figures 9 and 11-13.

Figures 18 and 19 present the combined effects of parameters of $b$ and Pr on the surface temperature, $\theta(0)$. Note that, the solutions of $\theta(0)$ are only acceptable if the obtained result is positive. It can be observed from Figure 18 that, the values of $\theta(0)$ correspond to $b=0.2$ and 1.0 are physically acceptable, when $\operatorname{Pr}$ are greater than its critical values of $\operatorname{Pr}_{c}=45.7509$ and 32.0921 respectively. Similarly, the possible solutions of positive values of $\theta(0)$ for $\operatorname{Pr}=0.72$ and 7 are obtained when $b$ are lower than 
its critical points of $b_{c}=\mathbf{0 . 6 5 5 8}$ and $\mathbf{1 . 9 5 3 5}$, respectively. These critical values could be used as a benchmark for other research works, specifically for those deals with the boundary condition of $\mathrm{NH}$.

\section{Conclusions}

The present study has discussed the steady two-phase flow of a dusty Casson model over a vertical stretching sheet. In addition, this study has also presented how the influences of modified magnetic field and buoyancy force together with boundary condition of $\mathrm{NH}$ affected the velocity and temperature profiles of fluid and dust phases, respectively. The significant findings from this study can be summarized as follows:

- The distributions of $f^{\prime \prime}(0)$ and $\theta(0)$ provide an important insight into the circumstances that happen on the surface of the sheet.

- The dust particle is found to be the reasoning factor, which decreases fluid transport and increases the fluid heating process, when $\beta$ is increased.

- The velocities of fluid and dust particles (temperature) decreases (increases) with the increase in $\alpha_{1}$ and $M$.

- The velocity (temperature) profile of fluid and dust phases increases (decreases) as Gr is increased.

- The temperature profile of both fluid and dust phases increases as $b$ increases. Conversely, a reverse trend is clearly depicted when Pr grows.

- The skin friction coefficient and Nusselt number possess the similar behavior under the effects of $\alpha_{1}, A, b$ and $\beta$. Meanwhile, an opposite trend in Nusselt number is noticed with the presence of Pr and Gr.

Author Contributions: Conceptualization, A.R.M.K., and N.S.A.; methodology, N.S.A., and S.M.Z.; software, N.S.A., and N.A.N.A.; validation, A.R.M.K., N.F.M., and N.S.A.; formal analysis, N.S.A.; investigation, A.R.M.K, M.Z.S., and N.S.A.; resources, D.L.C.C., S.S., N.A.N.A., and M.Z.S.; writing-original draft preparation, N.S.A; writing-review and editing, A.R.M.K.; supervision, A.R.M.K. and M.Z.S.; funding acquisition, A.R.M.K. All authors have read and agreed to the published version of the manuscript.

Funding: The authors gratefully acknowledge Universiti Malaysia Pahang for the financial support through Matching grant UMP-UTP-UTM-IIUM under RDU 182306.

Conflicts of Interest: The authors declare no conflict of interest.

\section{Nomenclature}

$\begin{array}{ll}(u, v)-\left(\mathrm{ms}^{-1}\right) & \text { components of fluid velocity along } x \text { and } y \text { direction } \\ T(\mathrm{~K}) & \text { fluid's temperature } \\ T_{p}(\mathrm{~K}) & \text { particle's temperature } \\ \mu_{B}\left(\mathrm{Nsm}^{-2}\right) & \text { plastic dynamic viscosity } \\ \rho\left(\mathrm{kgm}^{-3}\right) & \text { fluid density } \\ \rho_{p}\left(\mathrm{kgm}^{-3}\right) & \text { dust particle density } \\ \alpha_{1}\left({ }^{\circ}\right) & \text { aligned angle } \\ \sigma\left(\mathrm{Sm}^{-1}\right) & \text { electric conductivity of fluid } \\ \tau_{v}=1 / k\left(\mu \mathrm{s}^{-1}\right) & \text { Particles's relaxation time } \\ k\left(\mathrm{Nm}^{-2}\right) & \text { drag forces } \\ c_{p}\left(\mathrm{JK}^{-1} \mathrm{Kg}^{-1}\right) & \text { specific fluid's heat } \\ c_{s}\left(\mathrm{JK}^{-1} \mathrm{Kg}^{-1}\right) & \text { specific dust's heat } \\ \gamma_{T}\left(\mathrm{H}^{2} \alpha_{\mathrm{m}}-1\right) & \text { thermal relaxation time } \\ B_{0}\left(\mathrm{Am}^{-1}\right) & \text { magnetic-field strength } \\ A=\mu_{B} \sqrt{2 \pi_{c}} / p_{y} & \text { Casson parameter } \\ g\left(\mathrm{~ms}^{-2}\right) & \text { gravity acceleration } \\ \beta^{*}\left(\mathrm{~K}^{-1}\right) & \text { thermal expansion } \\ v\left(\mathrm{~m}^{2} \mathrm{~s}^{-1}\right) & \text { kinematic viscosity } \\ h_{s}\left(\mathrm{Wm}^{-2} \mathrm{~K}^{-1}\right) & \text { heat transfer coefficient }\end{array}$




\section{References}

1. Khalid, A.; Khan, I.; Khan, A.; Shafie, S. Unsteady MHD free convection flow of Casson fluid past over an oscillating vertical plate embedded in a porous medium. Eng. Sci. Technol. Int. J. 2015, 18, 309-317. [CrossRef]

2. Vajravelu, K. Convection heat transfer at a stretching sheet with suction or blowing. J. Math. Anal. Appl. 1994, 188, 1002-1011. [CrossRef]

3. Vajravelu, K.; Hadjinicolaou, A. Convective heat transfer in an electrically conducting fluid at a stretching surface with uniform free stream. Int. J. Eng. Sci. 1997, 35, 1237-1244. [CrossRef]

4. Merkin, J. Natural-convection boundary-layer flow on a vertical surface with Newtonian heating. Int. J. Heat Fluid Flow 1994, 15, 392-398. [CrossRef]

5. Lesnic, D.; Ingham, D.B.; Pop, I. Free convection boundary-layer flow along a vertical surface in a porous medium with Newtonian heating. Int. J. Heat Mass Transf. 1999, 42, 2621-2627. [CrossRef]

6. Chaudhary, R.; Jain, P. An exact solution to the unsteady free-convection boundary-layer flow past an impulsively started vertical surface with Newtonian heating. J. E. Phys. Thermophys. 2007, 80, 954-960. [CrossRef]

7. Kasim, A.; Mohammad, N.; Aurangzaib, S.S. Natural convection boundary layer flow of a viscoelastic fluid on solid sphere with Newtonian heating. World Acad. Sci. Eng. 2012, 64, 628-633. [CrossRef]

8. Salleh, M.; Nazar, R.; Pop, I. Numerical solutions of free convection boundary layer flow on a solid sphere with Newtonian heating in a micropolar fluid. Meccanica 2012, 47, 1261-1269. [CrossRef]

9. Kasim, A.; Mohammad, N.; Anwar, I.; Shafie, S. MHD effect on convective boundary layer flow of a viscoelastic fluid embedded in porous medium with Newtonian heating. Recent Adv. Math. 2013, 4, 182-189.

10. Mohamed, M.K.A.; Salleh, M.Z.; Nazar, R.; Ishak, A. Stagnation point flow over a stretching sheet with Newtonian heating. Sains Malays 2012, 41, 1467-1473.

11. Kasim, A.; Shafie, S. Mixed Convection Boundary Layer of a Viscoelastic Fluid past a Circular Cylinder with Constant Heat Flux. In Proceedings of the 1st Regional Conference on Applied and Engineering Mathematics 1, Eastern \& Oriental Hotel, Penang, Malaysia, 2-3 June 2010; pp. 124-129.

12. Kasim, A.; Mohammad, N.; Shafie, S. Unsteady MHD mixed convection flow of a micropolar fluid along an inclined stretching plate. Heat Transf. Asian Res. 2013, 42, 89-99. [CrossRef]

13. Ellahi, R.; Zeeshan, A.; Shehzad, N.; Alamri, S.Z. Structural impact of kerosene- $\mathrm{Al}_{2} \mathrm{O}_{3}$ nanoliquid on MHD Poiseuille flow with variable thermal conductivity: Application of cooling process. J. Mol. Liq. 2018, 264, 607-615. [CrossRef]

14. Ellahi, R.; Fetecau, C.; Sheikholeslami, M. Recent Advances in the Application of Differential Equations in Mechanical Engineering Problems. Math. Probl. Eng. 2018, 2018, 1584920. [CrossRef]

15. Ellahi, R.; Zeeshan, A.; Hussain, F.; Abbas, T. Study of Shiny Film Coating on Multi-Fluid Flows of a Rotating Disk Suspended with Nano-Sized Silver and Gold Particles: A Comparative Analysis. Coatings 2018, 8, 422. [CrossRef]

16. Ellahi, R.; Alamri, S.Z.; Basit, A.; Majeed, A. Effects of MHD and slip on heat transfer boundary layer flow over a moving plate based on specific entropy generation. J. Taibah Univ. Sci. 2018, 12, 476-482. [CrossRef]

17. Ellahi, R.; Hassan, M.; Zeeshan, A. Shape effects of spherical and nonspherical nanoparticles in mixed convection flow over a vertical stretching permeable sheet. Mech. Adv. Mater. Struct. 2017, 1231-1238. [CrossRef]

18. Ellahi, R.; Tariq, M.H.; Hassan, M.; Vafai, K. On boundary layer nano-ferroliquid flow under the influence of low oscillating stretchable rotating disk. J. Mol. Liq. 2017, 229, 339-345. [CrossRef]

19. Ellahi, R.; Raza, M.; Akbar, N.S. Study of peristaltic flow of nanofluid with entropy generation in a porous medium. J. Porous Media 2017, 20, 461-478. [CrossRef]

20. Kasim, A.M.; Zarith, S.O.; Shafie, S.; Pop, I. Generalized Blasius problem for a viscoelastic fluid with viscous dissipation and suction/injection effects. Int. J. Numer. Methods Heat Fluid Flow 2013, 23, 1242-1255. [CrossRef]

21. Kasim, A.R.M.; Jiann, L.Y.; Shafie, S.; Ali, A. The effects of heat generation or absorption on MHD stagnation point of Jeffrey fluid. AIP Conf. Proc. 2014, 1605, 404-409. [CrossRef]

22. Khan, Z.; Rasheed, H.U.; Abbas, T.; Khan, W.; Khan, I.; Baleanu, D.; Sooppy Nisar, K. Analysis of Eyring-Powell Fluid Flow Used as a Coating Material for Wire with Variable Viscosity Effect along with Thermal Radiation and Joule Heating. Crystals 2020, 10, 168. [CrossRef] 
23. Khan, U.; Zaib, A.; Khan, I.; Baleanu, D.; Nisar, K.S. Enhanced heat transfer in moderately ionized liquid due to hybrid $\mathrm{MoS}_{2} / \mathrm{SiO}_{2}$ nanofluids exposed by nonlinear radiation: Stability analysis. Crystals 2020, 10, 142. [CrossRef]

24. Lund, L.A.; Omar, Z.; Khan, I.; Baleanu, D.; Sooppy Nisar, K. Triple Solutions and Stability Analysis of Micropolar Fluid Flow on an Exponentially Shrinking Surface. Crystals 2020, 10, 283. [CrossRef]

25. Zokri, S.M.; Arifin, N.S.; Mohamed, M.K.A.; Salleh, M.Z.; Kasim, A.R.M.; Mohammad, N.F. Influence of radiation and viscous dissipation on magnetohydrodynamic Jeffrey fluid over a stretching sheet with convective boundary conditions. Mal. J. Fund. Appl. Sci. 2017, 13, 279-284. [CrossRef]

26. Abdelsalam, S.; Bhatti, M. Anomalous reactivity of thermo-bioconvective nanofluid towards oxytactic microorganisms. Appl. Math. Mech.-Engl. Ed. 2020, 41,711-724. [CrossRef]

27. Abumandour, R.M.; Eldesoky, I.M.; Kamel, M.H.; Ahmed, M.M.; Abdelsalam, S.I. Peristaltic thrusting of a thermal-viscosity nanofluid through a resilient vertical pipe. Z. Naturforsch. A 2020, 75, 727-738. [CrossRef]

28. Sadaf, H.; Abdelsalam, S.I. Adverse effects of a hybrid nanofluid in a wavy non-uniform annulus with convective boundary conditions. RSC Adv. 2020, 10, 15035-15043. [CrossRef]

29. Abdelsalam, S.I.; Bhatti, M. The study of non-Newtonian nanofluid with hall and ion slip effects on peristaltically induced motion in a non-uniform channel. RSC Adv. 2018, 8, 7904-7915. [CrossRef]

30. Saffman, P. On the stability of laminar flow of a dusty gas. J. Fluid Mech. 1962, 13, 120-128. [CrossRef]

31. Datta, N.; Mishra, S. Boundary layer flow of a dusty fluid over a semi-infinite flat plate. Acta. Mech. 1982, 42, 71-83. [CrossRef]

32. Ramesh, G.; Gireesha, B.; Bagewadi, C. MHD flow of a dusty fluid near the stagnation point over a permeable stretching sheet with non-uniform source/sink. Int. J. Heat Mass Transfer 2012, 55, 4900-4907. [CrossRef]

33. Manjunatha, P.; Gireesha, B.; Prasannakumara, B. Effect of radiation on flow and heat transfer of MHD dusty fluid over a stretching cylinder embedded in a porous medium in presence of heat source. Int. J. Appl. Comput. Math. 2017, 3, 293-310. [CrossRef]

34. Prakash, O.; Kumar, D.; Dwivedi, Y. Heat transfer in MHD flow of dusty viscoelastic (Walters' liquid model-B) stratified fluid in porous medium under variable viscosity. Pramana J. Phys. 2012, 79, 1457-1470. [CrossRef]

35. Gireesha, B.; Mahanthesh, B.; Gorla, R.S.R.; Krupalakshmi, K. Mixed convection two-phase flow of Maxwell fluid under the influence of non-linear thermal radiation, non-uniform heat source/sink and fluid-particle suspension. Ain Shams Eng. J. 2018, 9, 735-746. [CrossRef]

36. Mahanthesh, B.; Gireesha, B. Thermal Marangoni convection in two-phase flow of dusty Casson fluid. Results Phys. 2018, 8, 537-544. [CrossRef]

37. Arifin, N.S.; Zokri, S.M.; Kasim, A.R.M.; Salleh, M.Z.; Arifin, N.A.N. Jeffrey fluid embedded with dust particles over a shrinking sheet: A numerical investigation. J. Adv. Res. Fluid Mech. Therm. Sci. 2020, 74, 196-209. [CrossRef]

38. Kasim, A.R.M.; Arifin, N.S.; Ariffin, N.A.N.; Salleh, M.Z.; Anwar, M.I. Mathematical model of simultaneous flow between Casson fluid and dust particle over a vertical stretching sheet. Int. J. Integr. Eng. 2020, 12, 253-260. [CrossRef]

39. Kasim, A.R.M.; Arifin, N.S.; Zokri, S.M.; Salleh, M.Z. The investigation of a fluid-solid interaction mathematical model under combined convective Jeffrey flow and radiation effect embedded Newtonian Heating as the thermal boundary condition over a vertical stretching sheet. Defect Diffus. Forum 2020, 399, 65-75. [CrossRef]

40. Bhattacharyya, K.; Hayat, T.; Alsaedi, A. Exact solution for boundary layer flow of Casson fluid over a permeable stretching/shrinking sheet. Z. Angew. Math. Mech. 2013, 10, 522-528. [CrossRef]

41. Siddiqa, S.; Hossain, M.A.; Saha, S.C. Two-phase natural convection flow of a dusty fluid. Int. J. Numer. Methods Heat Fluid Flow 2015, 25, 1542-1556. [CrossRef]

42. Hussanan, A.; Salleh, M.Z.; Khan, I.; Shafie, S. Analytical solution for suction and injection flow of a viscoplastic Casson fluid past a stretching surface in the presence of viscous dissipation. Neural Comput. Appl. 2018, 29, 1507-1515. [CrossRef]

43. Makinde, O. Similarity solution of hydromagnetic heat and mass transfer over a vertical plate with a convective surface boundary condition. Int. J. Phys. Sci. 2010, 5, 700-710. [CrossRef]

44. Gangadhar, K.; Reddy, N.B.; Kameswaran, P. Similarity solution of hydromagnetic heat and mass transfer over a vertical plate with convective surface boundary condition and chemical reaction. Int. J. Nonlin. Sci. 2012, 13, 298-307. 
45. Seshadri, R.; Munjam, S.R. Mixed convection flow due to a vertical plate in the presence of heat source and chemical reaction. Ain Shams Eng. J. 2016, 7, 671-682. [CrossRef]

46. Mukhopadhyay, S. Casson fluid flow and heat transfer over a nonlinearly stretching surface. Chin. Phys. $B$ 2013, 22, 074701. [CrossRef]

47. Isa, S.M.; Ali, A.; Shafie, S. Stagnation point flow of MHD dusty fluid toward stretching sheet with convective surface. J. Teknologi 2016, 78, 95-100. [CrossRef]

48. Ramesh, G.; Gireesha, B.; Gorla, R.S.R. Boundary layer flow past a stretching sheet with fluid-particle suspension and convective boundary condition. Heat Mass Transfer 2015, 51, 1061-1066. [CrossRef]

49. Vajravelu, K.; Prasad, K.; Vaidya, H.; Basha, N.Z.; Ng, C.-O. Mixed convective flow of a Casson fluid over a vertical stretching sheet. Int. J. Appl. Comput. Math. 2017, 3, 1619-1638. [CrossRef]

C 2020 by the authors. Licensee MDPI, Basel, Switzerland. This article is an open access article distributed under the terms and conditions of the Creative Commons Attribution (CC BY) license (http://creativecommons.org/licenses/by/4.0/). 